\title{
Theoretical models for single-molecule DNA and RNA experiments: from elasticity to unzipping
}

\author{
S. Cocco ${ }^{\text {a }}$, J.F. Marko ${ }^{\mathrm{b}}$, R. Monasson ${ }^{\mathrm{c}}$ \\ a CNRS-Laboratoire de Dynamique des Fluides Complexes, 3 rue de l'Université, 67000 Strasbourg, France; \\ ${ }^{b}$ Department of Physics, The University of Illinois at Chicago, 845 W. Taylor St., Chicago, IL 60607; \\ c CNRS-Laboratoire de Physique Théorique de l'ENS, 24 rue Lhomond, 75005 Paris, France.
}

\begin{abstract}
We review statistical-mechanical theories of single-molecule micromanipulation experiments on nucleic acids. First, models for describing polymer elasticity are introduced. We then review how these models are used to interpret single-molecule force-extension experiments on single-stranded and double-stranded DNA. Depending on the force and the molecules used, both smooth elastic behaviors and abrupt structural transitions are observed. Third, we show how combining the elasticity of two single nucleic acid strands with a description of the base-pairing interactions between them explains much of the phenomenology and kinetics of RNA and DNA 'unzipping' experiments.
\end{abstract}

\section{INTRODUCTION}

Single-molecule studies that provide information on properties of one or a few interacting biomolecules are becoming increasingly important in biophysics. The precision of control and quantitative measurement, and simple interpretation of these experiments, make detailed theoretical analyses appropriate. The theory of single molecule micromanipulation experiments is a new development of polymer physics, emphasizing the structural richness of biopolymers (inhomogeneity of sequence, sequence-specific monomer interactions, transformations of secondary structure...). Both equilibrium and non-equilibrium aspects of single-molecule experiments reveal new basic physical problems.

This review presents some of the theoretical ideas that have been useful for description of single-molecule micromanipulation studies of nucleic acids. First, models useful for describing biopolymer elasticity will be presented. We will then review how these models are used to interpret single-molecule DNA force-extension experiments, which show both smooth elastic behaviors and abrupt structural transitions. Third, we will show how combining the elasticity of two single nucleic acid strands with a description of the base-pairing interactions between them explains much of the phenomenology of RNA and DNA 'unzipping' experiments.

The theoretical studies that we review use a wide range of tools and concepts from statistical mechanics and quantum mechanics. Single molecules are composed of a large number of elementary units (monomers). The nearest-neighbour character of the interactions between monomers often leads to partition functions with the form of path integrals (the curvilinear coordinate plays the role of time) which can be analyzed using the tools of quantum mechanics. Ideas from the theory of phase transitions are also extensively employed, for example to describe the abrupt, first-order-like structural changes frequently observed in stretching experiments. The kinetics of such transitions are thus related to problems from non-equilibrium statistical mechanics.

\section{THEORETICAL MODELS OF FLEXIBLE POLYMERS}

A number of polymer models have been used to model single-molecule experiments. Here we focus on applications relevant to double-stranded DNA, which is important biologically and also nearly ideal as an object for theoretical study.

\section{A. Gaussian Model}

The simplest description of a polymer is the Gaussian polymer (GP) model [1,2], which essentially considers a polymer to be a series of particles joined by Hookean springs (Fig. 1). Let us call $\mathbf{r}_{n}=\left(x_{n}, y_{n}, z_{n}\right)$ the location of monomer $n$. The vector leading from monomer $n-1$ to monomer $n, \mathbf{r}_{n}-\mathbf{r}_{n-1}$, obeys a Gaussian distribution of average zero and variance $<\left(\mathbf{r}_{n}-\mathbf{r}_{n-1}\right)^{2}>=b^{2}$, 


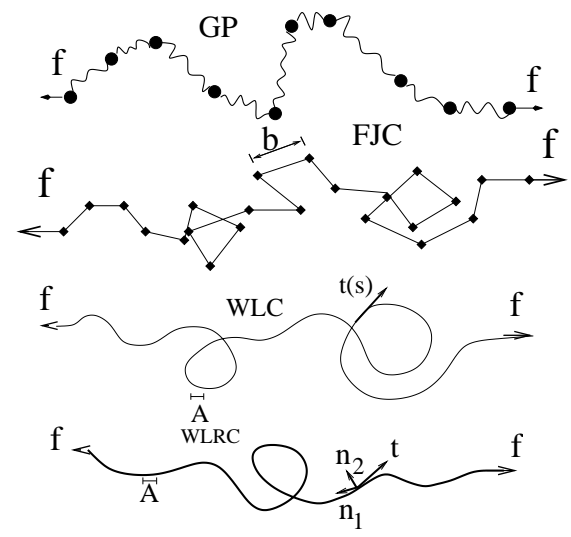

FIG. 1. Mathematical representations of polymeric chains. GP: the Gaussian polymer is made of $N$ monomers represented by harmonic springs. FJC: the Freely Jointed Chain is composed of $N$ bonds of fixed length $b$, with no correlation between the orientation of adjacent segments. WLC: the Worm Like Chain is a continuous model, characterized by a persistence length $A$; the orientation of the chain tangent $\mathbf{t}(s)$ is changing appreciably over contour lengths greater than $A$. WLRC: the Worm Like Rod Chain is described by a rotating three-dimensional coordinate system, with local triedron $\left(\mathbf{t}, \mathbf{n}_{1}, \mathbf{n}_{2}\right)$, along the curvilinear coordinate $s$.

$$
D\left(\mathbf{r}_{n}-\mathbf{r}_{n-1}\right)=\left(\frac{3}{2 \pi b^{2}}\right)^{3 / 2} \exp \left(-\frac{3\left(\mathbf{r}_{n}-\mathbf{r}_{n-1}\right)^{2}}{2 b^{2}}\right)
$$

where the index $n$ runs from 0 to $N$.

When submitted to a force $f$ along the $z$ direction, the Hamiltonian thus has a Gaussian elastic term, and a force-distance term,

$$
H_{G P}=\sum_{n=0}^{N} \frac{3 k_{B} T}{2 b^{2}}\left(\mathbf{r}_{n}-\mathbf{r}_{n-1}\right)^{2}-f\left(z_{N}-z_{0}\right)
$$

where the force is taken to act in the z-direction. The statistics of the z-component of the end-to-end vector are easily computed; for example the average end-to-end distance as a function of the force is

$$
<z>_{G P}=\frac{N b^{2}}{3 k_{B} T} f
$$

The Gaussian polymer as a whole behaves as a Hookean spring of zero rest length and stiffness $C=3 k_{B} T / N b^{2}$ proportional to the temperature and inversely proportional to the length $N$. This effective elasticity is a model for the entropic elasticity resulting from the decrease in the number of a polymer's configurations as is it extended. This basic picture of flexible polymer elasticity is the basis of rubber elasticity and a starting point for polymer physics [2].

\section{B. Freely-Jointed Chain model}

The GP has the unphysical feature that it can be indefinitely extended, and is therefore useful only for weakly stretched polymers, and even then only when physio-chemical details of the monomers are not of interest. A model which corrects the indefinite extensibility problem but which is still elementary and in wide use is the Freely Jointed Chain model [1,2], which consists of $N$ bonds of fixed length $b$ (the Kuhn length), allowed to point in any direction independently of each other (see Fig (1). When under zero force, the mean-squared end-to-end distance is $R^{2}=N b^{2}$, the familiar result for a random walk of $N$ steps.

When this chain is subjected to a force $f$, the bonds tends to align along the force, as dipoles in an electric field, with an energy

$$
H_{F J C}=-f b \sum_{n=1}^{N} \cos \theta_{n}
$$


where $\theta_{n}$ is the angle between the force and the $n^{\text {th }}$ bond directions. Since the segment orientations are decoupled, the partition function is easily calculated. The mean average end to end distance when a force $f$ is applied is

$$
<z>_{F J C}=N b<\cos \theta>=N b\left[\operatorname{coth}\left(\frac{f b}{k_{B} T}\right)-\frac{k_{B} T}{f b}\right]
$$

The small force behavior coincides with that of the GP expression (3). However, (5) departs from the GP at large forces, since the FJC model properly takes into account that the extension of the molecule cannot exceed the total contour length $N b$. For large $f,\langle z\rangle_{F J C} /[N b] \approx 1-k_{B} T /[b f]$.

\section{Worm-Like Chain}

The Worm-Like Chain (WLC) [1] is a continuous model without the sharp bends of the FJC. The chain is described by its unit tangent vector $\mathbf{t}(\mathbf{s})$, as a function of contour length $s$ along the chain. If no forces are applied, the tangent vector is presumed to undergo Gaussian fluctuations with zero mean and variance $\left\langle(d \mathbf{t} / d s)^{2}\right\rangle=1 / A$ (Fig. 1). The energy for this model in presence of a force $f$, is given by

$$
H_{W L C}=k_{B} T \frac{A}{2} \int_{0}^{L} d s\left(\frac{\partial \mathbf{t}}{\partial s}\right)^{2}-f \int_{0}^{L}(\mathbf{z . t}(s)) d s
$$

The first term is curvature energy that accounts for the resistance of the chain to bending, and the second term is the stretching energy due to application of the external force $f$. The partition function of a chain of length $L$, with tangent vectors at extremities $\mathbf{t}(s=0)=\mathbf{t}_{0}, \mathbf{t}(s=L)=\mathbf{t}_{1}$, can be written as a path integral,

$$
Z\left(L, f, \mathbf{t}_{0}, \mathbf{t}_{1}\right)=\int D \mathbf{t} e^{-H_{W L C} / k_{B} T}
$$

The significance of the parameter $A$ is made clear by the expression of average scalar product between tangent vectors at coordinates $s$ and $s^{\prime}$ at zero force,

$$
<\mathbf{t}(s) \cdot \mathbf{t}\left(s^{\prime}\right)>=\exp \left(-\left|s-s^{\prime}\right| / A\right)
$$

Therefore $A$ represents the characteristic distance above which tangent vectors decorrelate; $A$ is called the persistence length.

For a long chain under zero tension the WLC mean-squared end-to-end distance is $R^{2}=2 A L$ for $L>A$ (the formula for general $L$ is often useful, see [2]). Therefore the unperturbed random coil properties of the WLC are equivalent to those of the FJC and GP if we make the identification $2 A=b$ and $L=N b$. The unstretched WLC on large scales becomes a random walk of $N=L /(2 A)$ steps each of length $b=2 A$.

From a physical point of view, the FJC represents $N$ uncorrelated dipoles in an electrical field, and the average orientation of one dipole at equilibrium is obtained by classical statistical mechanics (eqns (1, 5). By contrast, the WLC describes, the "time" evolution of a dipole with moment of inertia $A$ in an electric field, with the role of time played by the contour length coordinate $s$. The introduction of the time dimension makes WLC equivalent to a quantum mechanical problem. The Schrödinger equation for the associated wave function can be analytically solved for small and large force limits, and can be numerically solved for general force [3,, 4 .

At small forces $<<k_{B} T / A$ the Hookean behavior (3) is recovered (i.e. with $b \rightarrow 2 A$ and $N \rightarrow L / b$ ) while for large forces $<z>_{W L C} / L \approx 1-\sqrt{k_{B} T /(4 A f)}$.

\section{ELASTICITY OF DOUBLE- AND SINGLE-STRANDED DNAS: EXPERIMENTS AND THEORY}

\section{A. Double-stranded DNA (dsDNA) under tension}

Reviews of experiments and theory on dsDNA elasticity can be found in $[5$, 8 . In Fig. 2 we report the force extension curve of a single dsDNA. Experimental data obtained by [14,24 for $\lambda$-DNA of length $48502 \mathrm{bp}$, or $L=16.4$

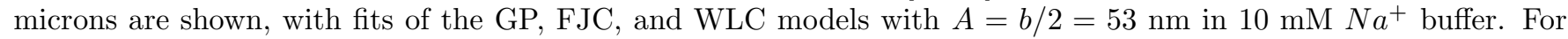
dsDNA, the Kuhn length $b=106 \mathrm{~nm}$ is much larger than the natural base pair spacing of $3.4 \AA$. dsDNA is not naturally described by the FJC model because consecutive bases, stacked onto each other, are not free to reorient 


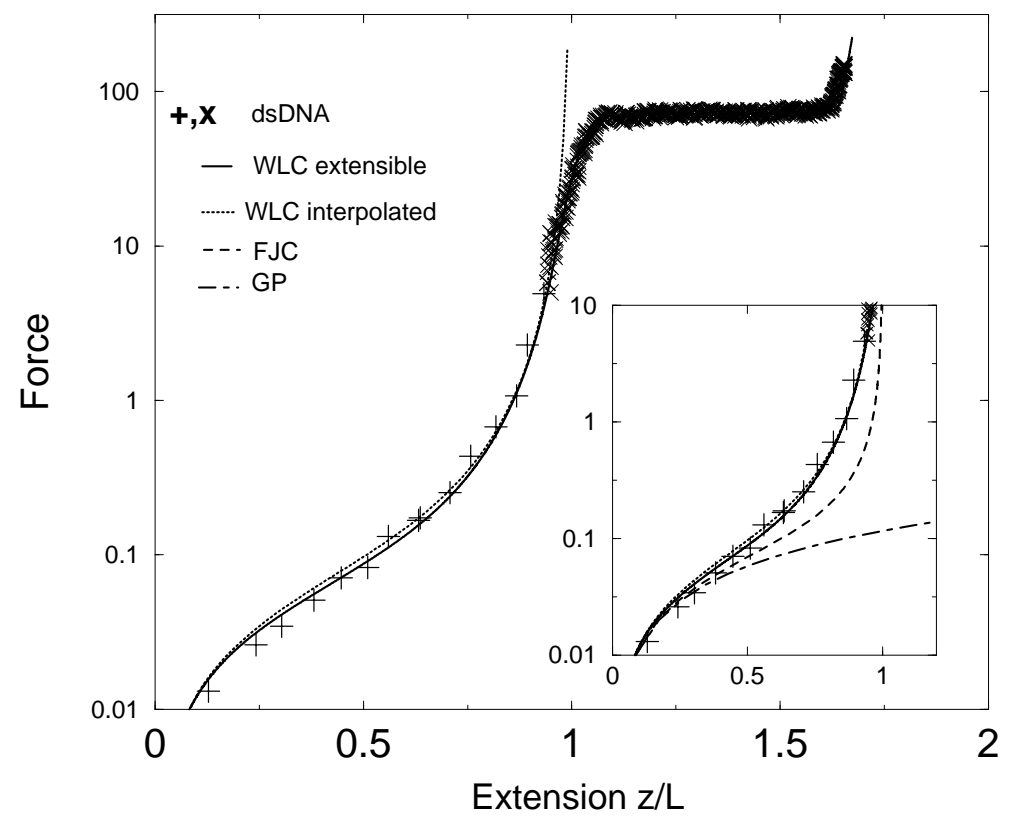

FIG. 2. Force-extension curve for a $\lambda$-dsDNA with equilibrium contour length $L=16.4 \mu \mathrm{m}$ in $10 \mathrm{mM} N a^{+}$buffer. Experimental data $(\mathrm{x})$ are from [24] and $(+)$ from [14]. The plateau at $f \simeq 70 \mathrm{pN}$ indicates the cooperative transition to SDNA. The extensible WLC model with persistence length $A=53 \mathrm{~nm}$, Young modulus $\gamma \approx 1000$ pN, and two states dsDNA-SDNA reproduces accurately the experimental behavior [23]. Marko and Siggia's interpolation formula [3] (9) is very accurate up to forces of $10 \mathrm{pN}$. Predictions from the GP and FJC $(b=2 A=106 \mathrm{~nm})$ models are plotted in the Inset.

independently of each other. The success of the WLC shows that dsDNA behaves as a semiflexible polymer, with a bending modulus $A k_{B} T$.

Several analytical interpolation formulae for the WLC, and modifications of the FJC introduced to fit accurately the data are discussed and compared in [7]10. Marko and Siggia proposed a simple interpolation formula that is close to the exact numerical solution of the force-extension response of the WLC [4, 34,

$$
f_{W L C}(z) \simeq \frac{k_{B} T}{A}\left[\frac{1}{4(1-z / L)^{2}}-\frac{1}{4}+\frac{z}{L}\right]
$$

This expression reduces to the exact solution as either $z \rightarrow 0$ or $z \rightarrow L$, but differs from the exact solution by up to $\approx 10 \%$ near $f=0.1 \mathrm{pN}$ (Fig 2). Bouchiat et al. [10] have introduced correction terms to eqn (9), in the form of a seventh order polynomial in $z / L$. The resulting approximation for $f_{W L C}(z)$ is accurate to $0.1 \%$. According to formula (3), a force $f_{0}=3 k_{B} T / b$ is required to extend dsDNA by a fraction of its contour length; from $b \simeq 100 \mathrm{~nm}$ we see that the characteristic force associated with the entropic elasticity of dsDNA is $f_{0} \approx 0.1 \mathrm{pN}$.

Fig $2 \mathrm{~B}$ shows that experimental data are well fitted by the FJC model for forces $f<0.1 \mathrm{pN}$, and by the WLC up to $f<5 \mathrm{pN}$. Various experiments analyzed in terms of the WLC give $A=50 \pm 5 \mathrm{~nm}$ in $10 \mathrm{mM} \mathrm{Na}$ [11, ]. The persistence length of DNA is reduced in high salt concentrations by electrostatic screening of the repulsive charge along the backbone; electrostatic effects have been taken into account in the WLC model by Barrat and Joanny through Debye-Huckel interactions [12, 1, 13, 7].

Fitting larger-force experimental data demands the introduction of the stretching elastic modulus of the molecule, $\gamma \simeq 1000 \pm 200 \mathrm{pN}$, quantitatively consistent with the relation between the bending modulus, $A k_{B} T$ and the Young modulus $Y=\gamma /\left(\pi R^{2}\right) \simeq 300 \mathrm{MPa}(R=10 \AA$ is the double helix radius $)$ for an elastic rod,

$$
A=\frac{\pi}{4 k_{B} T} Y R^{4} \equiv \frac{\gamma R^{2}}{4 k_{B} T}
$$

The value of the elastic modulus of DNA indicates that thermal fluctuations of the axial base pair distance $h$ of the order of $\left\langle(h-<h>)^{2}>\simeq k_{B} T /(\gamma<h>) \simeq 0.14 \AA\right.$. This order of magnitude is in agreement with molecular dynamics simulations, providing a consistent picture of the elasticity at the atomic and mesoscopic scale [17]. Axial vibrational modes have been studied in [18] and compared to Raman spectroscopy measurements. 


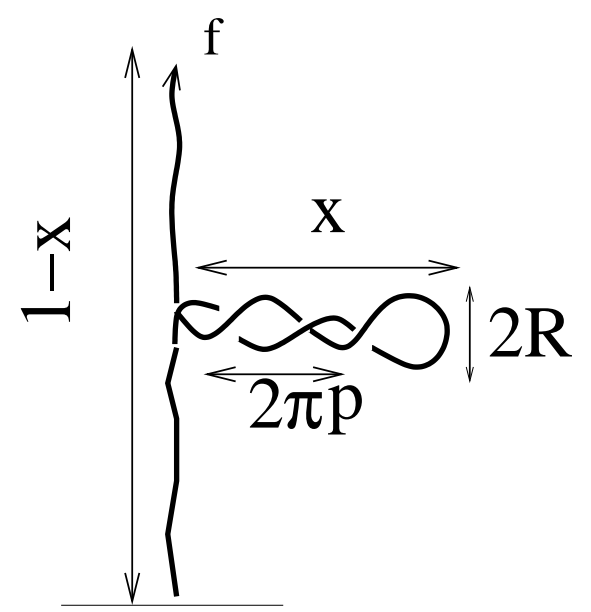

FIG. 3. Phase coexistence in a supercoiled DNA under tension. A fraction $x$ of the length is plectonemic supercoil with radius $R$ and pitch $P$, while the remaining fraction $1-x$ is in an extended conformation.

When dsDNA molecule is subjected to a force of $f=65 \mathrm{pN}$ it undergoes a structural transition to another conformation, S-DNA, with a contour length 1.7 times larger than its B-DNA counterpart [9, 11, 14. Numerical investigations of the structure of S-DNA have been performed by Lavery and collaborators 14.15]. The force plateau around $f=65 \mathrm{pN}$ corresponds to a highly cooperative transition, reminiscent of a first order phase transition. A two state model proposed by Cluzel et al. [14]16], inspired from models introduced in the context of thermally-induced denaturation [19 21], is able to reproduce the B-to-S transition. A recent study has suggested that the extended S state is actually strand-separated with the $\mathrm{S}$ phase described as stretched ssDNAs [58].

In the simplest two-state model of the $\mathrm{B}$ to $\mathrm{S}$ transition, the molecule is described as a chain of $N$ elements (base pairs), which can be in states B (energy $E_{B}$, length $l_{B}$ ) or $\mathrm{S}$ (energy $E_{S}$, length $l_{S}>l_{B}$ ). Each element is associated a spin variable, $s=1$ (respectively -1 ) for the $\mathrm{B}$ (resp. S) state. The energy of the chain reads,

$$
H=\sum_{i=1}^{N}\left(E_{s_{i}}-f l_{s_{i}}\right)+\frac{\omega}{2} \sum_{i=1}^{N-1}\left(1-s_{i} s_{i+1}\right)
$$

$\omega$ represents a 'domain wall' energetic cost of a B-S frontier. Up to an additive constant,

$$
H=-\frac{\omega}{2} \sum_{i=1}^{N-1} s_{i} s_{i+1}-\frac{1}{2}(\Delta E-f \Delta l) \sum_{i=1}^{N} s_{i}
$$

where $\Delta E=E_{S}-E_{B}$ and $\Delta l=l_{S}-l_{B}$. Notice that eqn (12) is simply the Hamiltonian of a one-dimensional Ising model with magnetic field $h=\Delta E-f \Delta l$. The extension is obtained from the derivative of the free energy with respect to the force $f$. Comparison with experiments allows to determine quantitatively the domain wall energy, $\omega \simeq 4 k_{B} T$ [16]. The extensible WLC including nonlinearities which define two states of extension provides a way to fit the force-extension curve over a wide range of forces $0.01<f<100 \mathrm{pN}$ [22].

\section{B. Supercoiled DNA under tension}

dsDNA differs from simpler polymers because it exhibits torsional and bending stiffness. Try to impose a twist to an elastic rod while keeping it extended and fixed at one end. Then, if you relieve the tension, an interwound structure called a plectoneme will appear, Fig 3 (twisted telephone cords often form plectonemic supercoils). Similarly, dsDNAs under sufficient torsional stress interwinds to form plectonemic supercoils. Formally, the over- or underwinding of DNA is measured by the change in double-helical linking number. This is often expressed as $\sigma$, the fractional change in linking number relative to that of relaxed dsDNA (one right-handed, or positive link per 10.5 base pairs). Supercoiling of DNA is of tremendous importance to eubacteria. For example, in E. coli all the DNA is held under torsional stress and is topologically constrained with $\sigma \approx-0.06$. 
The elasticity of a single supercoiled DNA molecule has been experimentally measured by Strick et al. 23, 24] and by Léger et al. [25]. A rich behavior was observed. At small forces the molecule responds to increasing positive or negative supercoiling by first having its conformations slightly chirally perturbed, and then by forming plectonemes with appreciable shortening of its length. At forces $f>0.5 \mathrm{pN}$, negative supercoiling is released through the opening of the double helix into denaturation bubbles. At forces $f>3 \mathrm{pN}$, positive supercoiling induces the formation of regions exhibiting a new conformation called P-DNA. The structure of P-DNA has been deduced by molecular modeling [26]; it is essentially characterized by its exposed bases. P-DNA can be thought of as two tightly interwound ssDNAs.

The theory of stretched supercoiled DNA was initiated by Marko and Siggia [27, 28], who considered phase coexistence of linear, plectonemic, and denatured DNA in different regions of a supercoiled molecule. The relative extensions of these portions are determined by the degree of supercoiling $\sigma$ and the stretching force $f$.

At small forces, a fraction $x$ of the molecule is in the plectonemic $(p)$ regime, whereas the remaining $1-x$ fraction is extended $(s)$ (Fig 3 ). The free energy per unit of length $\mathcal{F}=F / L$ reads,

$$
\mathcal{F}(\sigma, z / L)=x \mathcal{F}_{p}\left(\sigma_{p}\right)+(1-x) \mathcal{F}_{s}\left(f, \sigma_{s}\right)
$$

$\sigma=\Delta L_{k} / L_{k}^{0}$ is the excess of density of supercoiling with respect to the rest configuration (dsDNA making a double helix turn in 10.4 bases, $\left.L_{k}^{0}=L / 10.4\right) ; \sigma$ is partitioned into extended and plectonemic regions: $\sigma=x \sigma_{s}+(1-x) \sigma_{p}$. The free energy of the extended phase equals the WLC free energy plus the twisting energy, $\mathcal{F}_{s}\left(f, \sigma_{s}\right)=\mathcal{F}_{W L C}(f)+$ $k_{B} T C L_{k}^{2} /\left(2 L^{2}\right)\left(2 \pi \sigma_{s}\right)^{2}$. $C$ is the twist persistence length known from experiment to be $C=75 \pm 30 \mathrm{~nm}$.

The free energy of the plectonemes is thus considered to be the sum of elastic (bending and twisting), electrostatic and entropic contributions, minimized over plectonemic parameters e.g. the pitch $P$ and radius $R$ (Fig 3). The entropic term represents the entropy lost by confining the DNA in the superhelix formation. The total free energy is obtained by minimization with respect to the plectonemic portion $x$. At large forces the structural transition to denatured DNA is also included in the model by allowing the plectonemic phase to be a mixture of denatured and normal plectonemic DNA. The theoretical force-extension curve at fixed supercoiling reproduces the experimental behavior 28, 23].

A semi-microscopic model has been used to describe thermal- and torque-induced denaturation in one phase diagram [29]. This work described the formation of denaturation bubbles when DNA is stretched at $f>0.5 \mathrm{pN}$ and negatively supercoiled. The critical torque at room temperature $\Gamma \approx-2 k_{B} T$ is in good agreement with the value inferred from the experiments by Strick et al. [24].

A generalization of the two-state Ising description (12) of the overstretching transition has been introduced by Sarkar et al. [30] to model the structural transition of a twisted and stretched DNA molecule observed in [25]. For each site five possible state are introduced: dsDNA, S-DNA, P-DNA, sc-PDNA (a supercoiled P state), and a left handed double helix Z-DNA. This last state, with a supercoiling degree $\sigma_{Z}=-1.3$, is proposed as an alternative to denatured ssDNA $(\sigma=-1)$. A force-torque diagram is derived that agrees with the experiments on the critical unwinding torque at zero force, $\simeq-2 k_{B} T$, and the torque to drive DNA into the $\mathrm{P}$ structure, $\simeq 7 k_{B} T$.

The elasticity of supercoiled DNA has also been studied at a more microscopic level. Twisting and bending deformations can be described by extending the WLC to include description of base-pair orientation using a triad of unit vectors (WLRC) (Fig 1) [31]. Moroz and Nelson [32], and Bouchiat and Mézard [33] have written the partition function of this model as a path integral in the space of the Euler angles parametrizing the orientations, limiting the integration measure to the paths with a fixed linking number $L_{k}$. The free energy is obtained from the ground state energy of a Schrödinger equation describing a particle moving on a unit sphere in the presence of electric and magnetic fields.

The WLRC model does not take into account self avoidance: the WLRC chain is a phantom chain that can pass through itself. The result is that linking number fluctuations are not well-defined in the continuum limit. This problem is reflected in a divergence of the ground state energy. This is strictly a technical problem since real DNA has self-avoidance interactions. To avoid this problem, Moroz and Nelson considered the unambiguous infinite force situation (fully stretched molecule), and obtained finite force results by means of perturbation theory. Bouchiat and Mezard have introduced a short distance cutoff (discretization of the chain) to suppress the singularity. Fitting the theory to experimental data [23], the twist persistence length $C$ can be determined but is largely dependent on the theoretical scheme followed: $C=120 \mathrm{~nm}$ is obtained by Moroz and Nelson, while $C=82 \mathrm{~nm}$ is obtained by Bouchiat and Mezard for a cutoff length of $7 \mathrm{~nm}$. 


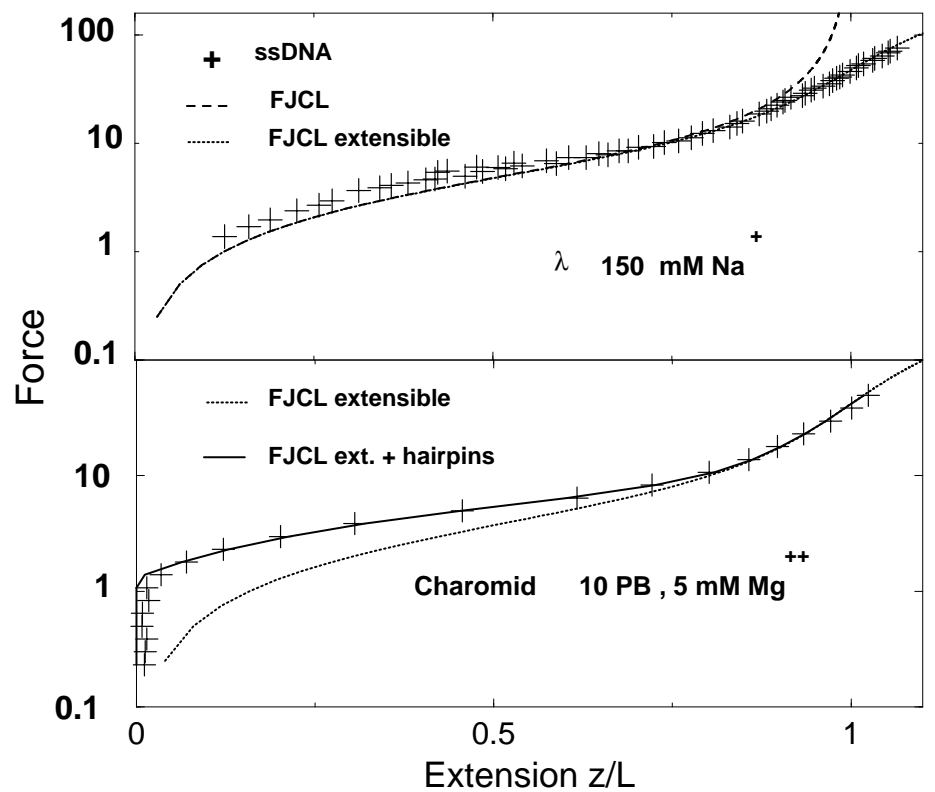

FIG. 4. Top: Force extension curve for a $\lambda$-ssDNA with equilibrium contour length $L_{s s}=l_{s s} N \equiv 0.56 \mathrm{~nm} \times 48502 \mathrm{bp}=27 \mu \mathrm{m}$ in $150 \mathrm{mM} \mathrm{Na}^{+}$. Experimental data $(+)$, dashed line: FJCL with $b=1.5 \mathrm{~nm}$, dotted line: extensible FJCL with $\gamma=800 p N$, from[11]. Bottom: Force extension curve for a Charomid ssDNA in $10 \mathrm{mM}$ phosphate buffer, $5 \mathrm{mM} \mathrm{Mg}^{++}$buffer, data are from [38], the fits are with the FJCL with $b=1.9 \mathrm{~nm}$ and $\gamma=800 \mathrm{pN}$ (dotted line) and with the hairpin model (full line) of $[41]$.

\section{Single-stranded DNA under tension}

Single-stranded DNA (ssDNA) is more flexible and can reach a larger extension per base pair than dsDNA. A sensible simple model of ssDNA is, at first sight, a FJC with a Kuhn length equal to the sugar-phosphate monomer backbone length $b=7 \AA$. However the ssDNA elasticity is complicated by nucleotide interactions, and as a result simple polymer models do not describe ssDNA elasticity over a wide range of forces.

For forces $f<20 \mathrm{pN}$ the experimental force extension curve for a 48502 base $\lambda$ ss-DNA in $150 \mathrm{mM} \mathrm{Na} a^{+}$has been fitted with a FJC-like (FJCL) model by Smith et al. [11] with two effective parameters: a Kuhn length $d=15 \AA$ and a contour length per base pair $l_{s s}=5.6 \AA$ that differs from the backbone distance (see Fig. 4 ). Note that due to the higher flexibility, the characteristic entropic force of the single strand, $f_{0}=3 k_{B} T / \sqrt{b l_{s s}} \approx 10 \mathrm{pN}$ (3), is much higher than for dsDNA. At forces $f>15 \mathrm{pN}$ the fit requires the introduction of a stretching modulus $\gamma=800 \mathrm{pN}$ (Section III A).

ssDNA elasticity depends strongly on salt concentration. At low salt $\left(1 \mathrm{mM} \mathrm{Na}^{+}\right.$, self avoidance interactions due to electrostatic self-repulsion along the charged sugar-phosphate backbone occurs. The experimentally observed logarithmic-like dependence of the extension upon force is well reproduced by Monte Carlo simulations [34.,35]. Electrostatic selfavoiding effects can be taken analytically into account using Barrat and Joanny formalism [36], or with a Hartree-Fock calculation from the WLC models [37].

At higher salt concentration (> $100 \mathrm{mM} \mathrm{Na}^{+}$, or in presence of $\mathrm{Mg}^{++}$) formation of secondary structure ('hairpins') by hydrogen bonding between complementary bases on the same strand strongly influences elastic properties. Experiments show that the force-extension behavior curve depends on the strand GC vs. AT content, and can be modulated using denaturing chemical agents that suppress hydrogen bonding [38, 34. A theoretical analysis of the elasticity of a polymer with hairpin secondary structure has been developed by Montanari and Mezard 42]. Conformations of hairpins taken into account are such that any two pairs of paired bases $(i<j, k<l)$ are independent $(i<j<k<l)$, or nested $(i<k<l<j)$ [39,40]. This representation do not include pseudoknots [41] but leads to a solvable recursion relation relating the partition functions of successively larger sequences [39], under the simplifying hypothesis that any two bases e.g. AT, GC, AG, ... have the same pairing free-energy. For an infinite molecule, a phase transition takes place between a folded (zero extension, $\left.f<f_{s}\right)$ and an extended phase $\left(f>f_{s}\right)$ with $f_{s}$ of the order of $1 \mathrm{pN}$, given reasonable choices of parameters (see Fig.4). 


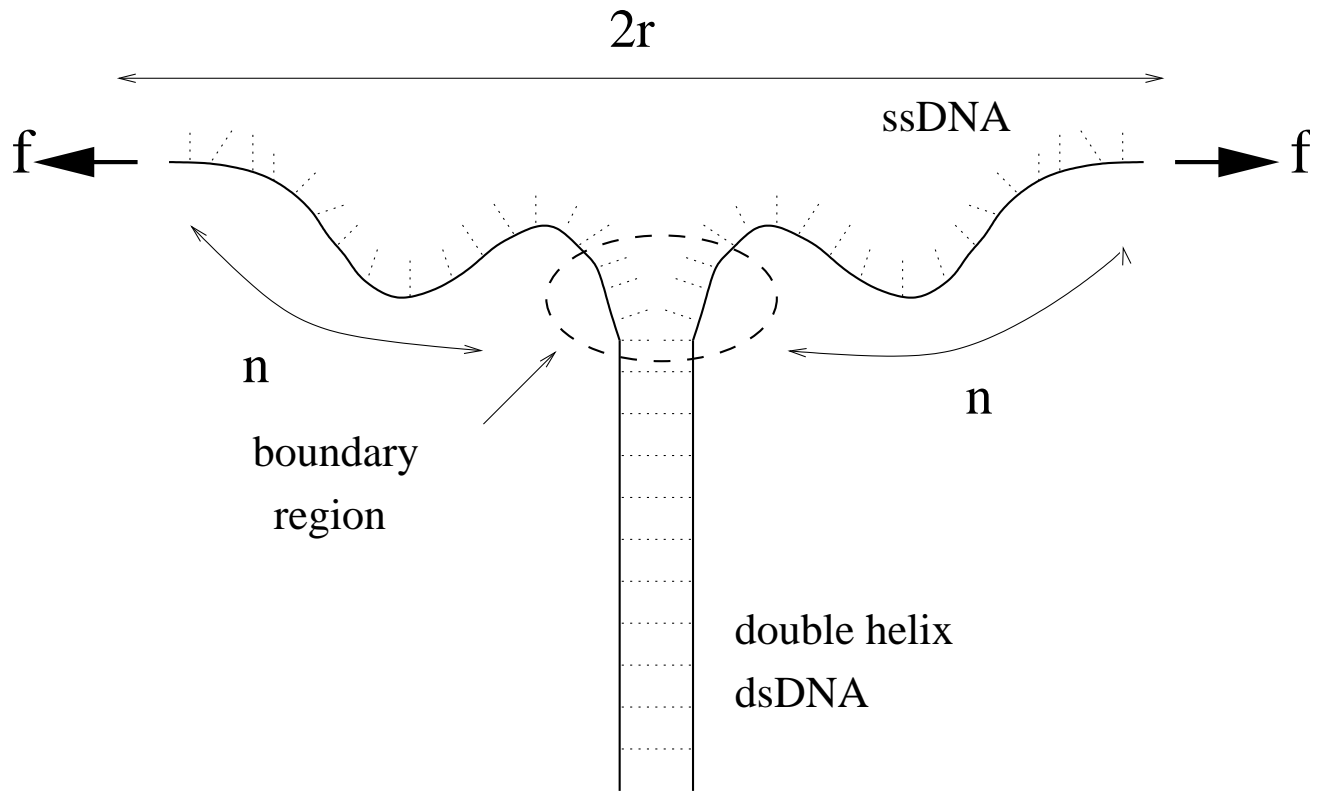

FIG. 5. Sketch of a DNA molecule with $n$ base pairs unzipped, as a result of a mechanical stress (applied force $f$ ). The distance between the two ssDNA ends is defined to be $2 r$.

\section{Elasticity of DNA in presence of DNA-folding proteins.}

In eukaryote cells, DNA is wrapped around octamers of histone proteins to form a more compact structure called a nucleosome. The long chromosomal DNAs of eukaryote cells are thus organized into long strings of nucleosomes, or 'chromatin fibers'. A single eukaryote chromosome may contain more than $10^{8}$ base pairs of DNA and roughly $10^{6}$ nucleosomes.

The elasticity of chromatin fiber has been experimentally studied by Cui and Bustamante 444. The experimental curves can be fitted with polymer models composed of units which, independently of each other, can be in a folded (short) or unfolded (long) state 443,36. These states are taken to correspond to stacked and unstacked nucleosomes. The elastic response of whole mitotic chromosomes can be related back to this fiber elastic response [36].

Very roughly, models of DNA folding by proteins will generally show a characteristic force at which the proteins will dissociate in equilibrium [43]. Given a free energy difference between the folded and unfolded states of $g$ per fold, and given an end-to-end length reduction of $d$, this characteristic force will be about $g / d$. Note that for large values of $d$ (e.g. by formation of large DNA loops, a common feature of gene-regulatory proteins) this implies low on-off equilibrium forces. It must be kept in mind that if the enthalpic component of the binding free energy is large, there may be large barriers for such loops to open and close, making equilibrium difficult to reach. Such situations should show theoretically interesting many-body thermal barrier-crossing kinetic phenomena.

\section{DNA AND RNA UNZIPPING}

Essevaz-Roulet, Bockelman and Heslot have shown that the two strands of a dsDNA can be pulled apart by a

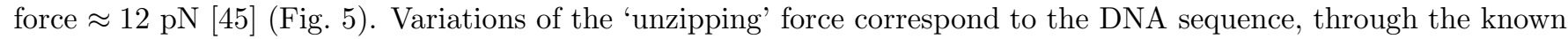
relationship between DNA sequence and base-pair interaction strengths 447. Higher forces were shown to correspond to DNA regions with higher GC densities, which in general have stronger base-pairing interactions than AT-rich sequences. Experimental unzipping force traces show a series of sawtooth signals attributed to stick-slip motion, with the sticking generated by DNA regions with higher GC content. This kind of experiment amounts to 'feeling' DNA sequence.

Current techniques are able to observe GC-content over long stretches of DNA $(\approx 10 \mathrm{~kb})$ with about 10 base pair resolution. It should be noted that it has been demonstrated that unzipping is sensitive to at least some single-base substitutions [59]. We now discuss some equilibrium and dynamical aspects of DNA and RNA unzipping. 


\section{A. Thermodynamics of DNA-RNA unzipping}

Control parameters for unzipping vary from experiment to experiment. Roughly speaking, either the force or the distance between strand extremities may be kept fixed (Fig. 5).

\section{Fixed pulling force}

With a fixed force $f$ on the molecule ends, the free energy $G$ of the molecule with $n$ base pairs opened is the difference between the free energy of the two extended single strands of $n$ bases each, and the free energy lost in unpairing the $n$ first base pairs $(i=1, \ldots, n)$ :

$$
G(f, n)=2 n \mathcal{F}_{s s}(f)-\sum_{i=1}^{n} g_{d s}(i)
$$

As discussed above, the free energy per base pair of stretched ssDNA, $\mathcal{F}_{s s}(f)$, can be expressed using the FJCL model for forces $f \simeq 10 \mathrm{pN}[9]$. At this high tension, nucleotide hairpin-formation effects are absent. Quadratic expansion of $\mathcal{F}_{s s}(f)$ around $f=10 \mathrm{pN}$ gives the free energy of a Gaussian polymer, $\mathcal{F}_{s s}^{G P}(f)=-f^{2} b^{2} /\left(6 k_{B} T\right)$ with an effective Kuhn length $b=7 \stackrel{\circ}{\text {. }}$

We start by considering an homogeneous sequence, where all base pairs have pairing free energy $g_{d s}=-g_{0}$. The unzipping critical force $f_{u}$ is simply given by the condition

$$
G(f, n) \equiv n g(f)=\left[2 \mathcal{F}_{s s}(f)+g_{0}\right] n=0
$$

For $f<f_{u}$, dsDNA is stable, and if $f>f_{u}$, the double helix unzips as in a first order phase transition. Using the Gaussian model for the ssDNA, we obtain $f_{u}^{G P}=\sqrt{3 k_{B} T g_{0}} / b$. The unzipping force of a homogeneous sequence is therefore directly related to the pairing free energy.

Rief et al measured the unzipping forces $f_{u}$ for DNAss of various repeated sequences [46]. It was found that $f_{u}($ poly dA- $\mathrm{dT})=9 \pm 3 \mathrm{pN}$ and $f_{u}\left(\right.$ poly dG-dC) $=20 \pm 3 \mathrm{pN}$, giving $g_{0}^{G C}(A-T)=0.8 k_{B} T, g_{0}^{F C L}(A-T)=1.1 k_{B} T$ $g_{0}^{G C}(G-C)=4.2 k_{B} T$, and $g_{0}^{F J C L}(G-C)=3.5 k_{B} T$ respectively. These values of the free energies of denaturation are compatible with thermodynamic data based on DNA melting 47]. It is to be noted that unzipping experiments give the only direct measurement of the relative free energies of ss and dsDNA at equal temperatures.

Unzipping has been discussed in the language of continuous phase transitions by Lubensky and Nelson 48,49. The unzipping free energy per base pair, $g(f)$, vanishes as $f_{u}-f$ with a discontinuous slope, as in a first order phase transition. However (as in interfacial wetting) as the unzipping transition is approached from below i.e. $f \rightarrow f_{u}^{-}$, the molecule the average number $\langle n>$ of open base pairs undergoes a continuous power-law divergence. From the probability to have $n$ open base pairs, $P(n)=g(f) /\left(k_{B} T\right) \exp \left[-(n g(f)) /\left(k_{B} T\right)\right]$, one obtains

$$
<n>=\frac{k_{B} T}{g(f)} \simeq \frac{1}{f_{u}-f}
$$

When the DNA molecule is subjected to a torque $\Gamma$, a torque-angle work contribution occurs in the unzipping free energy,

$$
g(f, \Gamma)=2 \mathcal{F}_{s s}(f)+g_{0}-\Theta_{0} \Gamma \quad .
$$

$\Theta_{0}=2 \pi / 10.4$ is the change in twist angle during conversion of dsDNA to separated strands [54]. The phase diagram for the unzipping transition in the force, torque plane is shown in Fig. 6 .

Along heterogeneous sequences, the free energy to open the first $n$ base pairs, $G(f, n)$ (14), can be calculated using the sequence dependent pairing free energy $g_{d s}(i)$ (e.g. from the Mfold server [50]). In Fig. 7, we show $G(f, n)$ for the RNA molecule called P5ab, mechanically unzipped by Liphardt et al. [51] with a force maintained fixed at the extremity through a feedback mechanism. The critical force is defined by the condition that the closed and open state have equal minimal free energies. Contrary to the homogeneous case, the free energy landscape at the critical force is not flat. It is characterized by high energy barriers $G^{*} \approx 10 k_{B} T$. The probability to have $n$ open base pairs is essentially zero if $n$ differs from the open and the closed configuration (Fig 7). Indeed, experiments show [51] that at the critical force the molecule essentially hops between open and closed configurations.

Lubensky and Nelson 48,49] have shown that the critical behavior around $f_{u}$ changes for large random sequence with respect to homogeneous sequences. Instead of the divergence $1 /\left(f_{u}-f\right)$ for the averaged number $<n>$ of base pairs, a stronger singularity $\left\langle n>\approx 1 /\left(f_{u}-f\right)^{2}\right.$ appears. 


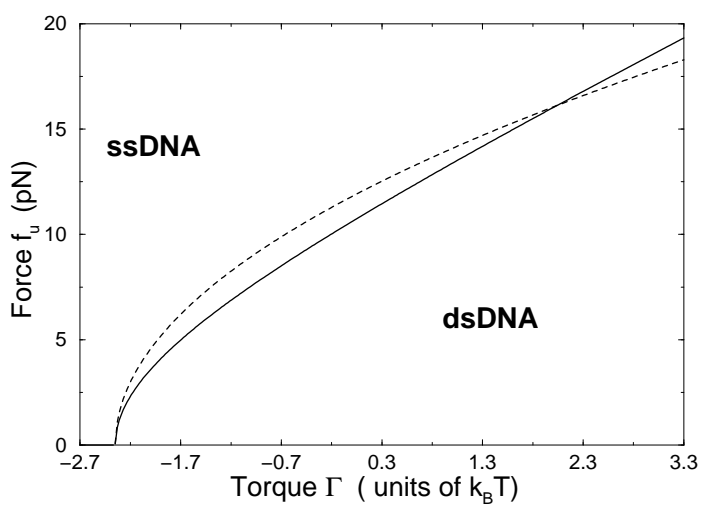

FIG. 6. DNA unzipping phase diagram as a function of torque $\Gamma$ in units of $k_{B} T$ and force $f$ in pN, from [54]. The solid line shows the results for the FJCL model of ssDNA elasticity, while the dashed line shows the result within the Gaussian approximation. At zero torque, the unzipping force is $f_{u} \simeq 12 \mathrm{pN}$; positive torque increase $f_{u}$, negative torques reduce $f_{u}$ until it vanishes at $\Gamma=-2.4 k_{B} T$.

\section{Fixed distance between extremities}

If the ssDNA ends are held apart at some distance $2 r$ (Fig. 5), some average number of bases $n$ will open. In the ideal case of a rigid opening device, the free energy cost to open $n$ base pairs is a sum of chain stretching and denaturation contributions,

$$
H_{d}(r, n)=W_{s s}(2 r, 2 n)-\sum_{i=1}^{n} g_{d s}(i)
$$

$W_{s s}(2 r, 2 n)$ is the work done by the force to stretch $2 n$ base pairs of ssDNA at a distance $2 r$. For simplicity we consider the GP free energy (3), considering as in the previous section the effective Kuhn length $b=7 \AA$, from the interpolation formula (9) [52], or from numerical inversion of the FJC extension vs. force (5) [53]. Thus, unzipping at fixed extension can bedescribed using

$$
W_{s s}^{G P}(2 r, 2 n)=3 k_{B} T \frac{r^{2}}{n b^{2}}
$$

The most probable value of the number of opened base pairs $\bar{n}$ is obtained by minimization of the free energy (18, 19) with respect to $n$. The number of unzipped based pairs is found to scale linearly with the distance, $\bar{n}(r)=r / d_{u}$ where $d_{u}=\sqrt{g_{0} / 3 k_{B} T} d=5 \AA$ is the projection of the monomer length along the force direction. The resulting free energy reads

$$
\mathcal{F}(r)=2 \sqrt{3 k_{B} T g_{0}} r / b=2 \bar{n}(r) g_{0}
$$

The tension $\bar{f}$ in the chain is simply the derivative of $\mathcal{F}$ with respect to $2 r, \bar{f}=\sqrt{3 k_{B} T g_{0}} / b=12 \mathrm{pN}$. This simple calculation shows that as unzipping proceeds quasi-statically, the ssDNA tension is a constant, just $f_{u}$. Note that the excess free energy per unpaired base for fixed extension is double the free energy of denaturation because the work done extending the ssDNAs adds to the work done when opening the molecule. This indicates a strategy to determine $g_{d s}$ unambiguously from unzipping force data.

The analysis of the fluctuations around the minimum free energy gives $\left\langle f-f_{u}\right\rangle=O\left(1 / r^{2}\right)$. Note that in the constant-force ensemble result, $f-f_{u} \approx 1 /<r>49$. The fixed-distance and fixed-force ensembles are equivalent only in the thermodynamic limit $r \rightarrow \infty$.

The unzipping force at small distance between extremities is sensitive to the detailed structure of the pairing potential as a function of the interbase distance. The semimicroscopic model introduced in [54,55] predicts a force barrier of $\simeq 300 \mathrm{pN}$ at a distance $2 r \simeq 21 \AA$, at which the hydrogen bond are broken but the bases are still stacked in the double helix configuration. This force barrier might be directly observable in an AFM experiment.

To take into account the experimental apparatus, Bockelmann et al. have included in their theory the effects of the two dsDNA linker arms of $N_{d s}$ base pairs and extension $r_{d s}$ ), and the cantilever stiffness [53,55]. The free energy (18) is then 

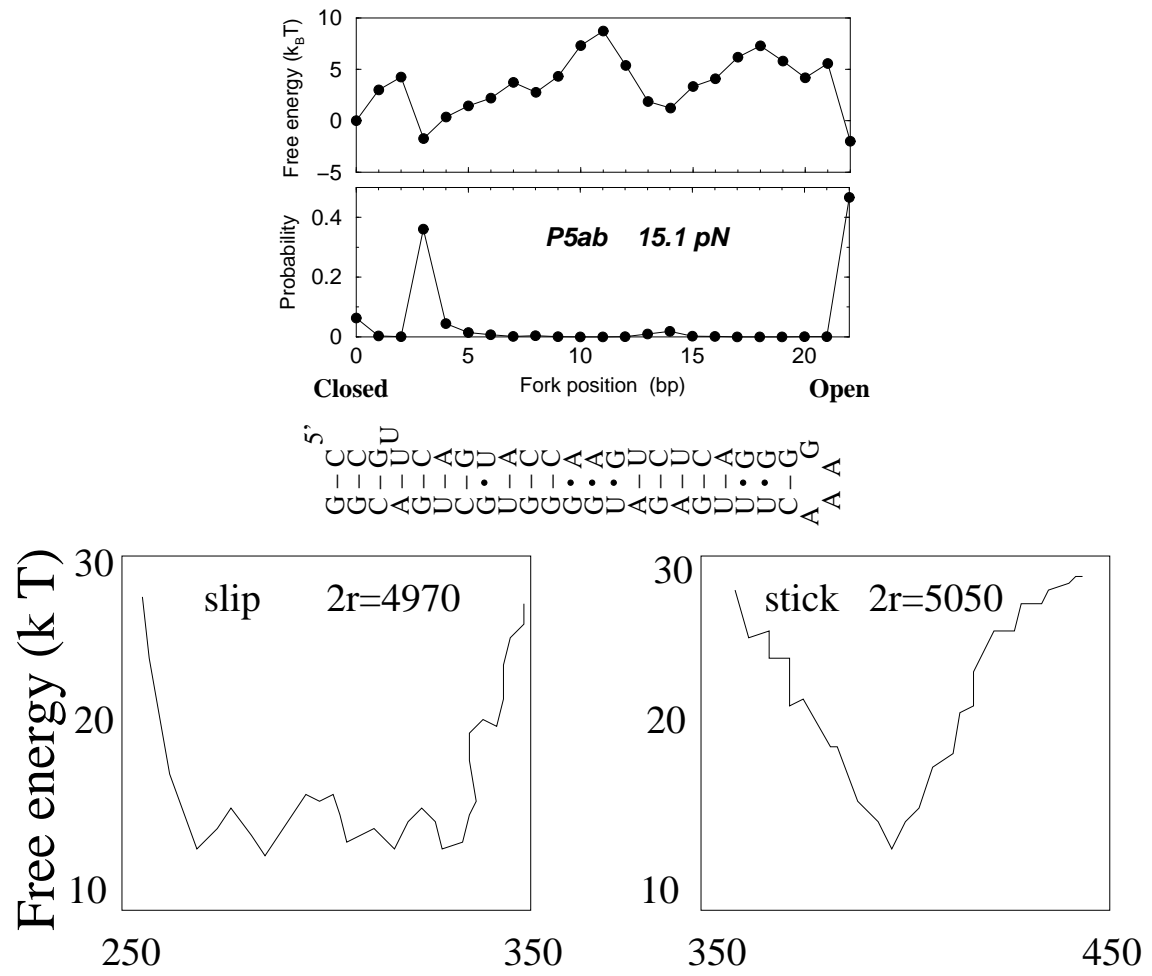

\section{fork position}

FIG. 7. Top: Free energy (in $k_{B} T$ ) and probability distribution of the opening fork at the critical force $f=15 \mathrm{pN}$ for the P5ab molecule (middle) from [59]. Bottom: Schematic representation of the free energy landscape for a displacement of 4970 $\mathrm{nm}$ (left, corresponding to slip phase in the force) and of $5050 \mathrm{~nm}$ (right, corresponding to the stick phase in the force) during the unzipping of a $\lambda$-DNA (from [58]).

$$
H_{d}^{c}\left(r, r_{s s}, r_{d s}, n\right)=W_{s s}\left(2 r_{s s}, 2 n\right)+W_{d s}\left(r_{d s}, N_{d s}\right)-\sum_{i=1}^{n} g_{d s}(i)-k_{\text {lever }}\left(r-2 r_{s s}-r_{d s}\right)
$$

The partition function is obtained by summing over all possibles value of $n, r_{s s}, r_{d s}$. The free energy $g_{d s}(i)$ can be computed using e.g. the Mfold program for base-pair interactions [50]. The ssDNA, dsDNA and the lever can be considered as three springs in series, with an effective stiffness is $k_{t o t}^{-1}=k_{s s}^{-1}+k_{d s}^{-1}+k_{l e v e r}^{-1}$. The spring constant of dsDNA, $k_{d s} \simeq 0.03 \mathrm{pN} / \mathrm{nm}$ for a dsDNA total length of 15000 bases in the experiment of Bockelmann et al., is obtained from the derivative of $f^{W L C}$ (including the Young modulus) calculated at a force of $12 \mathrm{pN}$. The ssDNA stiffness is $k_{s s} \simeq 6 k_{B T} /\left(b^{2} n\right) \simeq 50 / n \mathrm{pN} / \mathrm{nm}$, and the cantilever stiffness equals $k_{\text {lever }}=0.25 \mathrm{pN} / \mathrm{nm}$. For less than $\approx 1500$ unzipped base pairs, $k_{t o t}$ essentially reduces to the dsDNA linker stiffness. When more bases are unzipped, the dominant contribution comes from the ssDNA stiffness.

The DNA sequence dependence results in a complicated free energy landscape that generates a 'stick-slip' variation of the force during unzipping [59]. The stick phase corresponds to the presence of one deep minimum, and the slip phase to a flat free energy landscape see Fig 6 . The analytical description of Bockelmann et al. predicts that the height $h$ of the potential barrier increases as $h \approx \delta^{2}$ with the fluctuations $\delta$ of the pairing free energy, and decreases $h \approx k_{\text {tot }}^{-1}$ with the effective stiffness.

\section{B. Kinetics of unzipping}

The kinetics of unzipping at short length scales is affected by the presence of barriers with various physical origins, which makes it an activated process. 


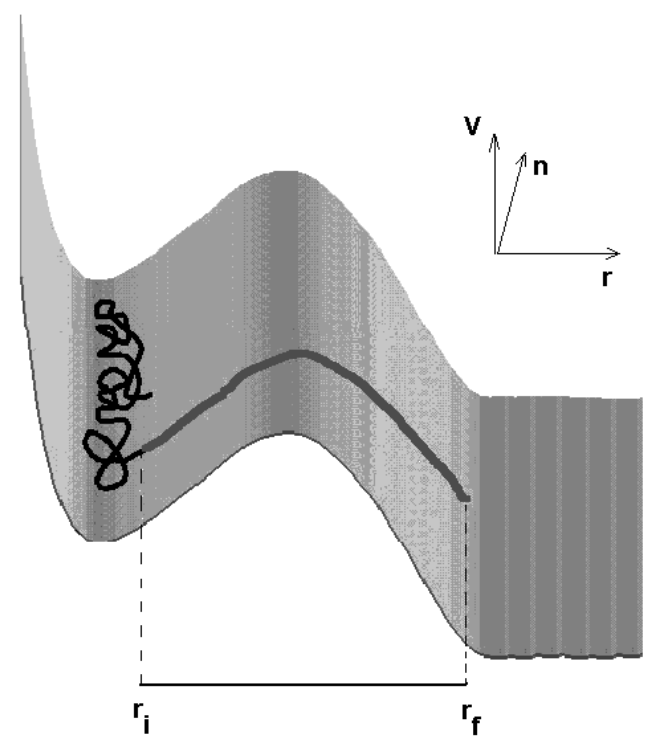

FIG. 8. Schematic representation of the saddle-point polymer conformation $r^{*}(n)$ which crosses the barrier of $V(r) ; r_{i}$ and $r_{f}$ are the radii of the edges of the nucleation bubble.

\section{Homogeneous sequences and unzipping initiation barriers}

Unzipping of homogeneous DNA requires the crossing of a barrier whose physical origin is the following. We imagine $r$, the half distance between the two bases of one pair to be a reaction coordinate indicating whether the pair is bonded $(r \simeq 10 \AA)$ or open $(r>11-12 \AA$ due to the very short range of H-bond). The effective free energy $V(r)$ of the base pair as a function of $r$ is shown in Fig. 8. It is low for both small (pairing energy) and large (entropy gain) values of $r$, and exhibits a maximum around $r \simeq 10.5 \AA$ where the H-bond is broken but bases are still stacked in the double helix conformation and are not free to move [54]. The set of half distances $r(n)$ between the two strands defines an abstract polymer (Fig. 8). At low enough forces this polymer is confined to the potential well (closed state). When a force $f$ larger than $f_{u}$ is applied at one extremity, the polymer escapes from the well (unzipping). As in a first order phase transition, nucleation theory can be employed to understand the opening kinetics [56].

The kinetics are slowed because of the activated crossing of the free energy barrier. A saddle-point calculation provides the optimal configuration of the polymer for crossing the barrier [54]. This configuration is made of a transition 'bubble' of a few $\simeq 4$ bases long, and free energy cost $G^{*}(f)$. The time of unzipping initiation grows exponentially with the activation free energy $G^{*}(f), t(f)=t_{0} \exp \left(G^{*} / k_{B} T\right)$. The elementary time $t_{0}$ corresponds to the time necessary for the polymer to escape from the saddle-point configuration along the unstable direction in the free energy landscape [56]. When the applied force is smaller than $f_{u}$, the molecule may still unzip. The opening time, which still depends on the barrier free energy, is now exponentially large in the equilibrium free energy $N g(f)$ where $N$ is the number of base pairs.

The determination of the opening time $t(f)$ at fixed force provides in turn the distribution of unzipping forces when the molecule is loaded with a fixed rate [57]. The most probable unzipping force exhibits a rich pattern depending on the loading rate and on the length of the sequence [54,55].

\section{Sequence-dependent barriers}

The above mechanism is mostly relevant in the kinetics of initiation of opening of the double helix. During unzipping of a long double helix, the sequence dependent free energy landscape of Fig 团 with barriers of $\approx 10 k_{B} T$ is responsible for a slow hopping dynamics between the open and closed states, i.e. the slip-stick behavior observed by Bockelmann et al 45,59 .

In experiments by Liphardt et al [51], small helix-loop RNA structures (essentially short regions of double-helical RNA terminated with a short loop) were held in such a way that equilibrium fluctuation between open and closed 
states occurred. The timescale observed was close to $1 \mathrm{sec}$ [51], remarkably long for a few-nm-long molecule. In [60] a dynamical model was introduced for the motion of the 'fork' separating the base paired and opened regions of the molecule, allowing computation of the opening and closing rate as a function of the force. The model, which describes the experimental data well, is based on the following elementary rates of opening and closing base pair $n$

$$
r_{0}(n)=r e^{-g_{0}(n) / k_{B} T}, \quad r_{c}(f, n)=r e^{-2 \mathcal{F}_{s s},(f, n)}
$$

The opening rate is taken to depend only on the pairing free energy since the short range hydrogen bond is broken before the force-length work over the longer $\approx 0.7 \mathrm{~nm}$ distance can be done. Conversely, to close the base pair, work must be first done against the applied force, and so the closing rate is taken to be depend only on the force. The separation of length scales of the range of base pair interaction and base pair extension after unzipping is thus used to justify placing most of the force-dependence in the zipping rate, with most of the interaction dependence in the unzipping rate. More sophisticated rate models will require further experiments to determine their form.

\section{CONCLUSION}

We have presented a very brief overview of the theory used to think about single-molecule nucleic acid micromanipulation experiments. The field of single-molecule experiments is evolving so rapidly at present that we have been forced to omit many exciting topics. Here we present a few general comments about what has been learned and suggest some directions that might be particularly interesting for study in the near future.

A feature common to all the studies described above, and to the theory of other types of single-biomolecule experiments, is the central role of statistical mechanics. The interaction of this field with statistical mechanics is fundamental: the understanding (in some cases, even the primary data analysis) of single-molecule DNA experiments requires statistical mechanics. Additionally, previously unimagined statistical-mechanical problems are resulting from the huge range of experimental possibilities for DNA and DNA-protein micromechanical experiments.

The first phase of single-DNA experiments involved basic characterization of dsDNA and ssDNA, and from the theoretical side involved development and solution of statistical-mechanical theories for the molecules subjected to stresses. The studies reviewed above essentially fall into this first class, and are characterized by a degree of quantitative success, thanks both to the efforts of experimentalists and theorists, which is unprecedented in soft condensed matter physics.

The second phase, which we are in at present, involves the study of modifications of the basic molecules, e.g. by unzipping, or by action of proteins acting on DNAs under mechanical control. The statistical mechanics of this second class of problems is less well developed, and includes problems far from thermal equilibrium such as DNAs acted on by processive, ATP-powered motor-like enzymes. The diversity of challenging and new statistical physics problems in this second class is mind-boggling. The second phase is also forcing theorists to confront the information content of nucleic acids since sequence plays an essential role in targeted nucleic acid-protein interactions.

The third class of problems involves applications of the lessons learned, to the description of cell machinery in vivo, or at least under in-vivo-like conditions. Such experiments are in their infancy, and extension of theoretical physics into this domain is still in a dark age. However, we can look forward to a time when we understand processes such as cell division and growth, gene regulation, and other cell biological functions in statistical-mechanistic terms. The lessons being learned now about the importance of statistical mechanical ideas to biochemical-micromechanical experiments on nucleic acids will thus become an important component of future quantitative understanding of cell biology.

Acknowledgements We thank A. Sarkar, V. Croquette and D. Bensimon for their helpful comments on this manuscript. This work was in part supported by the NSF(USA; JM and SC: DMR-9734178, RM: DMR-9808595), the Research Corporation (JM), the A. della Riccia Foundation (SC), and by the Focused Giving Program of Johnson and Johnson (JM).

[1] P.J. Flory, Statistical Mechanics of Chain Molecules. Hanser, Munich (1989).

[2] M. Doy, S.F. Edwards, The Theory of Polymer Dynamics. Oxford University Press, Oxford (1986).

[3] C. Bustamante, J.F. Marko, E.D. Siggia, S. Smith, Entropic elasticity of lambda-phage DNA. Science 265, 1599 (1994). 
[4] J.F. Marko, E.D. Siggia, Stretching DNA. Macromolecules 28, 209 (1995).

[5] R.H. Austin, J.P. Brody, E.C. Cox, T. Duke, W. Volkmuth, Stretch genes. Physics Today 50, 32 (Feb. 1997).

[6] C. Bustamante, S.B. Smith, J. Liphardt, D. Smith, Single-molecule studies of DNA mechanics, Curr. Op. Struct. Biol 10, 279 (2000).

[7] M.D. Wang, H. Yin, R. Landick, J. Gelles, S. Block, Stretching DNA with optical tweezers, Biophys. J. 72, 1335 (1997).

[8] T. Strick, J.F. Allemand, V. Croquette, D. Bensimon, Twisting and stretching single DNA molecules, Progress in Biophysics and Molecular Biology 74, 115 (2000).

[9] S.B. Smith, L. Finzi, C. Bustamante, Direct mechanical measurements of the elasticity of single DNA molecules by using magnetic beads. Science 258, 1122 (1992).

[10] C. Bouchiat, M.D. Wang, J F. Allemand, T. Strick, S.M. Block, V. Croquette, Estimating the persistence length of a worm-like chain molecule from force extension measurements, Biophys. J. 76, 409 (1999).

[11] S. Smith, Y. Cui, C. Bustamante, Overstretching B-DNA: The elastic response of individual double-stranded and singlestranded DNA molecules. Science 271, 795 (1996).

[12] J.L. Barrat, J.F. Joanny, Persistence length of polyelectrolytes chains, Europhys. Lett. 24, 333 (1993).

[13] C.G. Baumann, S.B. Smith, V.A. Bloomfield, C. Bustamante, Ionic effects on the elasticity of single DNA molecules. Proc. Natl. Acad. Sci. (USA) 94, 6185 (1997).

[14] P. Cluzel, A. Lebrun, C. Heller, R. Lavery, J.L. Viovy, D. Chatenay, F. Caron, DNA: An extensible molecule. Science 271, 792 (1996).

[15] A. Lebrun, R. Lavery, Modelling extreme deformations of DNA, Nucl. Acids Res. 24,2260 (1996).

[16] P. Cizeau, J.L. Viovy, Modelling extreme extension of DNA, Biopolymers 42, 383-385 (1997).

[17] J. Marko, M. Feig, B.M. Pettitt, Unification of the microscopic atomic fluctuations with mesoscopic elasticity of the DNA double helix, preprint (2001).

[18] S. Cocco, R. Monasson, Theoretical study of collective modes in DNA at ambient temperature, J. Chem. Phys. 112, 10017 (2000).

[19] T.L. Hill, J. Chem. Phys. 30, 383 (1959).

[20] B. Zimm, J. Bragg, J. Chem. Phys. 31, 526 (1959).

[21] Y. Kafri, D. Mukamel, L. Peliti, Why is the DNA denaturation transition first order? Phys. Rev. Lett. 85, 4988 (2000).

[22] J.F. Marko, DNA under high tension: overstretching, undertwisting, and relaxation dynamics, Phys. Rev. E 57, 2134 (1998).

[23] T.R. Strick, J-F. Allemand, D. Bensimon, A. Bensimon, V. Croquette, The elasticity of a single supercoiled DNA Molecule, Science 271, 1835 (1996).

[24] T.R. Strick, J.F. Allemand, D. Bensimon, V. Croquette, Behavior of supercoiled DNA, Biophys. J. 74, 2016 (1998).

[25] J-F. Léger, G. Romano, A. Sarkar, J. Robert, L. Bourdieu, D. Chatenay, J.F. Marko, Structural Transitions of a Twisted and Stretched DNA Molecule, Phys. Rev. Lett. 83, 1066 (1999).

[26] J.F. Allemand, D. Bensimon, R. Lavery, V. Croquette, Stretched and overwound DNA forms a Pauling-like structure with exposed bases, Proc. Natl. Acad. Sci. (USA) 74, 2016 (1998).

[27] J.F. Marko , E.D. Siggia, Statistical mechanics of supercoiled DNA. Phys. Rev. E 52, 2912 (1995).

[28] J.F. Marko, Supercoiled and braided DNA under tension, Phys. Rev. E 55, 1758 (1997).

[29] S. Cocco, R Monasson, Statistical mechanics of torque induced denaturation of DNA, Phys. Rev. Lett. 83, 5178 (1999).

[30] A. Sarkar, J-F. Léger, D. Chatenay, J.F. Marko, Structural transitions in DNA driven by external force and torque, Phys. Rev. E 63, 051903 (2001).

[31] B. Fain, J. Rudnick, S. Ostlund, Conformations of linear DNA, Phys. Rev. E 55, 7364 (1997).

[32] J.D. Moroz, P. Nelson, Torsional directed walks, entropic elasticity, and DNA twist stiffness, Proc. Natl. Acad. Sci. (USA) 94, 1441 (1997).

[33] C. Bouchiat, M. Mézard, Elasticity model of a supercoiled DNA molecule, Phys. Rev. Lett. 80, 1556 (1998).

[34] M.N. Dessinges, B. Maier, M. Peliti, D. Bensimon, V. Croquette, Stretching single stranded DNA, a real self avoiding and interacting heteropolymer. preprint (2001).

[35] Y. Zhang, H. Zhou, Z.C. Ou-Yang, Stretching single-stranded DNA: interplay of electrostatic, base-pairing, and base-pair stacking interactions, Biophys. J. 81, 1133 (2001).

[36] S. Cocco, R. Monasson, J. Yan, A. Sarkar, J.F. Marko, Elastic response of folding polymers, preprint (2002).

[37] C. Bouchiat, Hartree-Fock computation of self avoiding flexible polymer elasticity, preprint (2001).

[38] B. Maier, D. Bensimon, V. Croquette, Replication by a single DNA-polymerase of a stretched single strand DNA. Proc. Natl. Acad. Sci. (USA) 97, 12002 (2000).

[39] R. Bundschuh, T. Hwa, Statistical mechanics of secondary structures fromed by random RNA sequences. Phys. Rev. E 65, 031903 (2002).

[40] A. Pagnani, G. Parisi, F. Ricci-Tersenghi, Glassy transition in a disordered model for the RNA secondary structure. Phys. Rev. Lett. 84, 2026 (2000).

[41] H. Isambert, E.D. Siggia, Modeling RNA folding paths with pseudoknots: Application to hepatitis delta virus ribozyme Proc. Natl. Acad. Sci. (USA) 97, 6515 (2000)

[42] A. Montanari, M. Mezard, Hairpin formation and elongation of biomolecules, Phys. Rev. Lett. 86, 2178 (2001).

[43] J.F. Marko, E.D. Siggia, Driving proteins off DNA using applied tension, Biophys. J. 73, 2173 (1997). 
[44] Y. Cui, C. Bustamante, Pulling a single chromatin fiber reveals the forces that maintain its higher-order structure. Proc. Natl. Acad. Sci. (USA) 97, 127-132 (2000).

[45] B. Essevaz-Roulet, U. Bockelmann, F. Heslot, Mechanical separation of the complementary strands of DNA, Proc. Natl. Acad. Sci. (USA) 94, 11935 (1997).

[46] M. Rief, H. Clausen-Schaumann, H.E. Gaub, Sequence-dependent mechanics of single DNA molecules, Nat. Struct. Biol. 6, 346 (1999).

[47] K. Breslauer, R. Frank, H. Blocker, L.A. Marky, Predicting DNA duplex stability from the base sequence, Proc. Natl. Acad. Sci. (USA) 83, 3746 (1986).

[48] D.K. Lubensky, D.R. Nelson, Pulling pinned polymers and unzipping DNA, Phys. Rev. Lett. 85, 1572 (2000).

[49] D.K. Lubensky, D.R. Nelson, Single molecule statistics and the polynucleotide unzipping transition, Phys. Rev. E 65, 031917 (2002).

[50] M. Zuker, Calculating nucleic acid secondary structure, Curr. Opin. Struct. Biol. 10, 303-310 (2000).

[51] J. Liphardt, B. Onoa, S.B. Smith, I. Tinoco JR, C. Bustamante, Reversible unfolding of single RNA molecules by mechanical force. Science 292, 733-737 (2001).

[52] E.R. Thompson, E.D. Siggia, Physical limits on the mechanical measurement of the secondary structure of Bio-molecules. Europhys. Lett. 31, 335-340 (1995).

[53] U. Bockelmann, B. Essevaz-Roulet, F. Heslot, DNA strand separation studied by single molecule force measurements. Phys. Rev. E 58, 2386 (1998).

[54] S. Cocco, R. Monasson, J.F. Marko, Force and kinetic barriers to unzipping of the DNA double helix Proc. Natl. Aca. Sci. (USA) 98, 8608-8613 (2001).

[55] S. Cocco, R. Monasson, J.F. Marko, Force and kinetic barriers to initiation of DNA unzipping. Phys. Rev. E 65, 041907 (2002).

[56] J.S. Langer, Statistical theory of the decay of metastable states. Ann. Phys. (N.Y.) 54, 258-275 (1967).

[57] E. Evans, K. Ritchie, Dynamic strength of molecular adhesion bonds. Biophys. J. 72, 1541-1555 (1997).

[58] I. Rouzina, V.A. Bloomfield, Force-induced melting of the DNA double helix. Biophys. J. 80, $882-893$ (2001).

[59] U. Bockelmann, P. Thomen, B. Essevaz-Roulet, V. Viasnoff, F. Heslot, Unzipping DNA with optical tweezers: high sequence sensitivity and force flips. Biophys J. 82, 1537-1553 (2002).

[60] S. Cocco, J.F. Marko J.F., R. Monasson, Slow nucleic acid unzipping kinetics from sequence-defined barriers. preprint (2002). 